2014·28(2):241-247

4. Yoshizato T, Dumitriu B, Hosokawa K, et al. Somatic mutations and clonal hematopoiesis in aplastic anemia. N Engl J Med. 2015;373(1):35-47

5. Palomo L, Meggendorfer M, Hutter S, et al. Molecular landscape and clonal architecture of adult myelodysplastic/myeloproliferative neoplasms. Blood. 2020;136(16):1851-1862.

6. Schischlik F, Kralovics R. Mutations in myeloproliferative neoplasms their significance and clinical use. Expert Rev Hematol. 2017;10(11):961-973

7. Valent P, Kern W, Hoermann G, et al. Clonal hematopoiesis with oncogenic potential (CHOP): separation from CHIP and roads to AML. Int J Mol Sci. 2019;20(3):789.

8. Meggendorfer M, Walter W, Haferlach T. WGS and WTS in leukemia: a tool for diagnostics? Best Pract Res Clin Haematol. 2020;33(3):101190.

9. Nadarajah N, Wagner A, Bejar R, et al. Creating a variant database for the American Society of Hematalogy by consensus variant clas- sification of common genes associated with hematologic malignancies. Blood. 2020;136(Suppl 1):4-5.

10. Radakovich N, Nagy M, Nazha A. Machine learning in haematological malignancies. Lancet Haematol. 2020;7(7):e541-e550

11. Matthijs G, Souche E, Alders M, et al. Guidelines for diagnostic nextgeneration sequencing. Eur J Hum Genet. 2016;24(1):2-5.

12. Jennings LJ, Arcila ME, Corless C, et al. Guidelines for validation of next-generation sequencing-based oncology panels: a joint consensus recommendation of the Association for Molecular Pathology and College of American Pathologists. J Mol Diagn. 2017;19(3):341-365.

13. Mangul S, Martin LS, Hill BL. Systematic benchmarking of omics computational tools. Nat Commun. 2019;10(1):1-11

14. Malcovati L, Galfi A, Travaglino E, et al. Clinical significance of somatic mutation in unexplained blood cytopenia. Blood. 2017;129(25):3371-3378.

15. Baer C, Pohlkamp C, Haferlach C, et al. Molecular patterns in cytopenia patients with or without evidence of myeloid neoplasm-a comparison of 756 cases. Leukemia. 2018; 32(10):2295-2298.

\title{
Recognition of thrombotic risk of thrombocytosis in iron deficiency
}

\author{
Hanny Al-Samkari, ${ }^{1}$ Craig M. Kessler ${ }^{2}$ and Michael Auerbach ${ }^{2}$ \\ ${ }^{1}$ Division of Hematology, Massachusetts General Hospital, Harvard Medical School, Boston, MA and 'Department of Medicine, \\ Georgetown University School of Medicine, Washington, DC, USA \\ E-mail: MICHAEL AUERBACH - mauerbachmd@abhemonc.com
}

doi:10.3324/haematol.2020.270496

I n 1999, Loo and Beguin examined the effect of recombinant human erythropoietin on megakaryopoiesis, noting that iron-replete and iron-overloaded rats treated with recombinant human erythropoietin demonstrated a one-third decreased duration of thrombocytosis compared with normal rats. ${ }^{1}$ The putative explanation given for the increased duration in the normal rats was erythropoietin-induced, iron-deficient erythropoiesis. This provocative hypothesis was subsequently supported by the findings of a post-hoc analysis ${ }^{2}$ of a large prospective study of oral versus intravenous or no iron as adjunctive therapy for erythropoiesis-stimulating agent-treated patients with cancer and chemotherapy-induced anemia. ${ }^{3}$ This study demonstrated that intravenous, but not oral, iron repletion resulted in marked improvements in hemoglobin and hematopoietic responses and also re-demonstrated the known associations of increased thromboses and thrombocytosis among patients with cancer receiving erythropoiesis-stimulating agents. Of 187 patients treated with an erythropoiesis-stimulating agent, 19 developed 29 venous thromboembolic events and those with platelet counts greater than 350,000 per microliter were three times more likely to develop venous thromboembolism. Notably, there was a reduced incidence of thrombocytosis and thrombotic complications in those treated with intravenous iron compared with those who were not.

Further corroborating evidence pointing to iron-restricted erythropoiesis resulting in reactive thrombocytosis and thrombosis was derived from a recently published study describing the rate, predictors, and thrombotic complications of thrombocytosis in patients with irondeficiency anemia. ${ }^{4}$ Utilizing queries of an institutional database containing clinical information on more than six million patients over a 40-year time period (1979-2019), supported by extensive manual chart reviews, thrombocytosis was observed in $32.6 \%$ of patients with iron-deficiency anemia, in whom $15.8 \%$ developed thrombotic complications. In contrast, thromboses occurred in $7.8 \%$ of those with iron deficiency but without thrombocytosis (Figure 1).

The etiological explanation for why iron-restricted erythropoiesis increases hypercoagulability has remained somewhat obscure. In this issue of Haematologica, Jimenez and colleagues describe an animal model of iron deficiency, using Sprague-Dawley rats, which could offer unique opportunities to explore multiple pathophysiological mechanisms for thrombocytosis and venous thrombogenesis. ${ }^{5}$ Furthermore, using sensitive histological and sophisticated high-frequency ultrasound techniques, the investigators were able to track the development and progression of thrombus formation. They were then able to correlate those changes with the generation of ex vivo biomarkers of platelet activation and indicators of global hemostasis. The potential use of this rat model to detect important targets that could be exploited therapeutically to mitigate the association of iron deficiency with thrombocytosis and thromboembolism is promising and encouraging.

In their elegant description of iron deficiency-induced augmentation of coagulability on thromboelastography and hemostasis following rat tail resection, prominent correlates of increased platelet adhesion and aggregation were clearly described. Using in vitro assays of platelet aggregation, the authors were able to postulate that not only is platelet activation an ongoing process in inflammatory conditions but in iron deficiency as well. Importantly, given the frequent comorbidity of iron defi- 
34,511 patients with Iron Deficiency Anemia CD-9 or ICD-10 coded diagnosis of iron deficiency anemia plus synchronous ferritin of $<30 \mathrm{ng} / \mathrm{mL}$

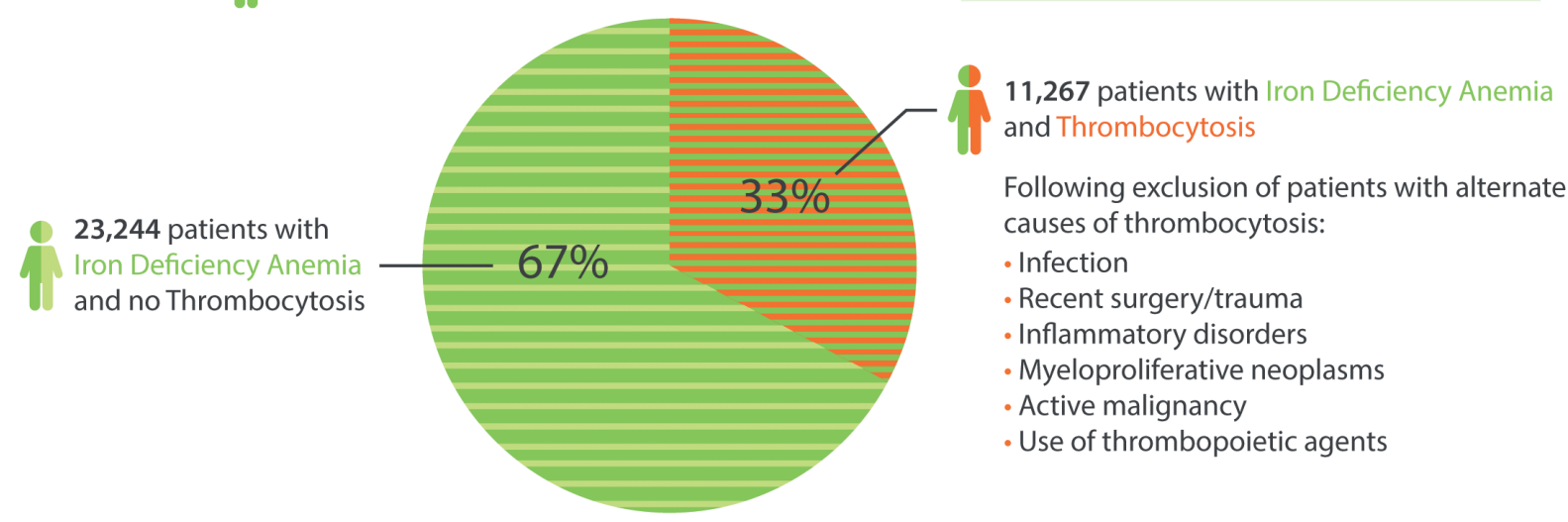

Thrombosis rate
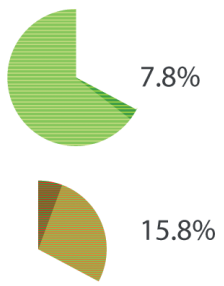

$15.8 \%$
Thrombotic events

Synchronous with iron deficiency anemia

Synchronous with both iron deficiency anemia and thrombocytosis
Types of thrombotic events

\section{- Arterial events: $0.7 \%$}

- Venous events: $7.1 \%$

- Arterial events: $1.4 \%$

- Venous events: $14.4 \%$

Figure 1. Rates of thrombocytosis and thrombosis among patients with iron-deficiency anemia. Rates of thrombocytosis and thrombosis in a recent large clinical database study of over six million patients, supported by extensive chart review to exclude alternative confounding causes of thrombocytosis and confirm thrombotic events. ${ }^{4}$

ciency with a number of clinical conditions with known thrombotic risk such as inflammatory bowel disease and cancer, this animal model clearly demonstrates that iron deficiency alone increases thrombotic tendency, consistent with the findings of aforementioned human studies. Perhaps even more importantly, treatment with ferric carboxymaltose (an intravenous iron formulation) reversed the prothrombotic changes in the rats, including thrombocytosis, changes in platelet function, and iron deficiency-associated increases in thrombus size in both venous and arterial models of thrombosis.

The development of a high quality animal model to lend support to the longstanding observation of iron deficiency mediated-thrombocytosis leading to a significantly higher risk of thrombosis is an important advance. Without stratifying for iron deficiency or its successful abatement by intravenous iron, the observation of a marked increment in thromboses in erythropoiesis stimulating agent-treated patients with cancer and chemotherapy-induced anemia and anemia associated with both non-dialysis and dialysis-dependent chronic kidney disease led to the near proscription of the use of a treatment which unqualifiedly leads to improvements in energy, activity and quality of life in a cohort with debilitating chronic illness. Despite a litany of prospective, randomized, placebo-controlled studies failing to show harm with the use of erythropoiesis-stimulating agents, especially in cancer and chemotherapy-induced anemia, ${ }^{6}$ extreme caution with their use remains the order of the day.
A now growing body of inferential evidence supports the use of intravenous iron as the preferred route of administration in most of the described conditions. This new animal model provides evidence that intravenous iron replenishment may have additional advantages in mitigating the thrombogenic potential of iron deficiency. It also suggests that intravenous iron might preferentially provide an important adjunctive therapy to mitigate the hypercoagulability associated with iron-deficiency states comorbid with multiple disorders in humans, such as cancer, inflammatory bowel disease, after bariatric surgery, abnormal uterine bleeding and pregnancy. Given that iron-deficiency anemia affects approximately $20 \%$ of the human population at any given point in time, ${ }^{7}$ even a modestly increased thrombotic risk imparted by iron deficiency has profound public health implications. Additionally, synergism of multiple thrombotic risk factors is well-documented, ${ }^{8}$ raising the specter of a potentially even greater thrombotic impact of this common problem given its frequent comorbidities. Thankfully, additional avenues of investigation to better understand these risks are open, as this animal model also suggests that critical biomarkers can now be explored and can then be correlated with objective in vivo events. Such biomarkers could also include the hypoxia-inducible factors HIF-1 and HIF-2, whose target genes include erythropoietin, the transferrin receptor and vascular endothelial growth factor. These targets are also associated with increased thrombogenesis.

Despite the overwhelming preponderance of published evidence supporting the safety and efficacy of intra- 
venous iron, ${ }^{9}$ reluctance to its use among practitioners prevails due to heightened concerns of danger fomented, at least in part, by misinformation and misinterpretation of the incidence and clinical nature of serious adverse events occurring in recipients of this treatment. ${ }^{10}$ The convincing evidence brought forth from these well-performed in vitro experiments and animal models provides information not only on a long-sought physiological mechanism for a commonly observed clinical event but more importantly on the need to heighten the awareness of a readily prescribed treatment modality which mitigates its occurrence.

\section{Disclosures}

HA-S: no conflicts of interest relevant to the present manuscript; consultancy (for Agios, Dova, Rigel and Argenx) and research funding (from Agios, Dova and Amgen). CMK: no relevant financial disclosures; $M A$ : research funding from AMAG Pharma (manufacturer of the IV iron ferumoxytol) and have done educational non-promotional talks for Pfizer and Pharmacosmos.

\section{Contributions}

Each of the authors contributed to the concept of the editorial, drafting the manuscript, critically revising it and designing the figure. All authors approved the final version.

\section{Acknowledgments}

HA-S is the recipient of the Harvard KL2/Catalyst Medical Research Investigator Training Award and an American Society of Hematology Scholar Award.

\section{References}

1. Loo M, Beguin Y. The effect of recombinant human erythropoietin on platelet counts is strongly modulated by the adequacy of iron supply. Blood. 1999;93(10):3286-3293.

2. Henry DH, Dahl NV, Auerbach MA. Thrombocytosis and venous thromboembolism in cancer patients with chemotherapy induced anemia may be related to ESA induced iron restricted erythropoiesis and reversed by administration of IV iron. Am J Hematol. 2012;87(3):308-310.

3. Henry DH, Dahl NV, Auerbach M, Tchekmedyian S, Laufman LR Intravenous ferric gluconate significantly improves response to epoetin alfa versus oral iron or no iron in anemic patients with cancer receiving chemotherapy. Oncologist. 2007;12(2):231-242.

4. Song $A B$, Kuter DJ, Al-Samkari $\mathrm{H}$. Characterization of the rate, predictors, and thrombotic complications of thrombocytosis in iron deficiency anemia. Am J Hematol. 2020 Jul 3. doi: 10.1002/ajh.25925. [Epub ahead of print].

5. Jimenez K, Leitner F, Leitner A, et al. Iron deficiency-induced thrombocytosis increases thrombotic tendency in rats. Haematologica. 2020;106(3):783-795

6. Gascon P, Nagarkar R, Smakal M, et al. A randomized, double-blind placebo-controlled, phase III noninferiority study of the long-term safety and efficacy of darbepoetin alfa for chemotherapy-induced anemia in patients with advanced NSCLC. J Thorac Oncol 2020;15(2):190-202.

7. Miller JL. Iron deficiency anemia: a common and curable disease. Cold Spring Harb Perspect Med. 2013;3(7):a011866

8. Pomp ER, le Cessie S, Rosendaal FR, Doggen CJ. Risk of venous thrombosis: obesity and its joint effect with oral contraceptive use and prothrombotic mutations. Br J Haematol. 2007;139(2):289-296.

9. Avni T, Bieber A, Grossman A, Green H, Leibovici L, Gafter-Gvili A. The safety of intravenous iron preparations: systematic review and meta-analysis. Mayo Clin Proc. 2015;90(1):12-23.

10. Auerbach M, Ballard H, Glaspy J. Clinical update: intravenous iron for anaemia. Lancet. 2007;369(9572):1502-1504. 